\title{
Robustness Management with Data Quality in Information Systems
}

\author{
Zhiting Song* and Yanming Sun \\ South China University of Technology \\ NO.381 Wushan Road, Guangzhou, Guangdong, China, 510641 \\ szt05_scut@163.com,meymsun@scut.edu.cn
}

\begin{abstract}
The data quality in information systems affects system robustness significantly Although a large body of research focuses on the issue of data quality, seldom has literature been done to develop effective control policies to manage system robustness associated with data errors. A process-oriented methodology is proposed to manage system robustness measured by performance, control cost and control time, which is achieved through establishing formal model of information systems and mathematical optimization models of system robustness. The proposed methodology captures how structural and functional characteristics of tasks affect system robustness and finds the optimal control policies, which facilitates the robusmess-based design and management of information systems respectively. The methodology is illustrgted in the case study.
\end{abstract}

Keywords: Robustness; Data Quality; Information Systems; Control

\section{Introduction}

Data quality has long been recognized âs an 1mportant issue in information systems [13]. Poor data quality often prevents mformation systems from yielding satisficing performance [4], which severely impacts system robustness. Although information systems have beenexensively studied in information system literature, there is a lack of research on system robustness associated with data errors. In reality, robustness is a desirable propery for informationsystems, while data errors have been the leading cause of business process interription in information systems [5]. It is necessary to develop effective control policies to ensure that systems are robust to data errors.

To maintain system robustness at a satisficing level in case of data errors, the paper first extends the yell-structured WF-net that Van der Aalst proposed via inserting dummy task transitions between two directly connected branches or merges to model information systems more formally. Based on the extended WF-net, the paper then incorporates structural and functional features of tasks into error control, and selects performance, control cost and control time related to data errors to measure robustness. Finally, it establishes optimization models to determine the optimal control policies that achieve target robustness on performance with maximal robustness on cost or maximal robustness on time. The paper provides a feasible and quantitative approach to robustness management for information systems exposed to the perturbation of data errors.

The paper expects to achieve the following objectives: (1) to model real-world information systems more formally, it extends the well-structured WF-net by introducing dummy task transitions; (2) to facilitate the robustness management of existing information systems, robustness optimization models are established to determine the optimal resource allocation policies that yield satisficing robustness on performance with maximal robustness on cost or robustness on cost time; (3) to provide guidance to the 
robustness-based design of information systems, the influence that structural and functional features of tasks exert on the optimal input levels of resource across tasks and error types is analyzed.

\section{Literature Review}

This article tries to develop effective control policies to make information systems robust to data errors. Three streams of literature are relevant to this paper: the first is related to data quality and data errors, the second is related to business process modeling, and the last is related to system robustness.

\subsection{Data Quality and Data Errors}

Data quality, originally identified as data accuracy, is a multidimensional concept beyond accuracy [6]. Although there is no uniform definition of data quality dimensions [7], the frequently mentioned dimensions are accuracy, completeness, consistency and timeliness which have been widely accepted in information system literatare [6] [8-9]. Data quality dimensions in this study refer to these four dimensions. The research of data quality dimensions provides a starting point to define data en or types [2]. For example, according to data quality dimensions of completeness, existence, valuation, presentation and disclosure, and rights and obligations in accomting literature, Lea et al. define five error types of completeness errors, existence errors, valuation errors, presentation and disclosure errors, and rights and obligations errors [10]. Similarly, error types in the paper are accuracy errors, completeness errors consistency errors and timeliness errors. Accuracy errors occur when the data are incorrect or even spurious. Completeness errors occur when all relevant information that should be contained does not fully exist. Consistency errors occur when data are not consistently represented, formatted or compatible with previous data. Timeliness errors occur when data are not disposed during a given period of time or processed in a timelymanner. Data errors mainly originate from system design and improper execution of tasks in the business processes of information systems, and this study focuses on the latter error source.

\subsection{Business Process Modeling}

Changes in the environments of information systems have made many information systems processes-oriente 111], which provides an idea to construct ontologies for information systems by business process modeling. There exist many methods for business process modeling. Flow chart is first introduced to model business processes, but it is unable to analyze properties of business processes [12]. Recently, workflow graph [13], event-driven process chains (EPC) [14], business process modeling notation (BPMN) and unified modeling language (UML) [4] also have been used to model business processes, but they all lack formal semantics. Petri nets (i.e., classical Petri nets and highlevel Petri nets) have been considered as powerful tools to model and analyze workflow [11]. Concretely speaking, Petri nets are not only applied to analyze behavior, atributes and events of workflow, but employed to model reachability, deadlock and other structural features of workflow [11] [15]. In a word, different methods can be applied to model business processes, but Petri nets are the only formal methods that can be used for both structural modeling and a wide range of qualitative and quantitative analysis [11] [16], which is vital to analyze robustness. A Petri net modeling a workflow process is called a Workflow net (WF-net), and the definition of well-structured WF-net is extended to specify the process-oriented ontology of information systems. 


\subsection{Robustness}

There is no agreement on the concept of robustness in information system literature. Tavana et al. define robustness as the ability to avoid failure caused by increasing complexity between people and technology in systems [17]. In reality, all perturbations apart from that complexity will influence robustness. Some researchers hold that a system is robust if it operates correctly with all perturbations inside and outside the system [1820]. It is undesirable if the normal operation consumes a lot of time or money. From the perspective of strategic management, Sawy et al. define robustness of information systems as the ability to yield satisficing performance under all environments quickly and inexpensively [21], which is adopted in the study for its comprehensiveness. To quantitatively analyze robustness, measures of robustness are proposed, and performance is a widely used metric of robustness [19-22]. Performance under a certain environment is computed either as the product of event probability and undesirable consequence related to the event [20] or key performance indexes of systems [22]. In the paper, performance is measured by the product of error probability and loss caused by data errors, which avoids subjectivity in selecting performance indexes. Intuitively, robust systems ean suffer perturbations at a low cost and in a short time. Thus, robustness can be measured from the perspectives of repair-recovery cost and repair-recovery time associated with perturbations [17] [23] which in the article are control cost and control ime associated with data errors. Moreover, standard deviation of system response [19], efficiency in switching to other configurations and the degree of variability of environmental conditions that systems can cope with are also said to be measures of robustness [21], but they are not frequently used.

In summary, above mentioned papets do not involve in robustness management associated with data quality, but provide essential elements to quantitative formulations of robustness management with data quality in information systems.

\section{Formal Model for Information Systems}

This section first proposes an extended well-structured WF-net to model information systems formally, and then describes the error propagation in the model.

\subsection{Business Process Modeling}

Definition 1. $P N=\left(R, T_{t} \cup T_{b} \cup T_{d} ; F\right)$ is an extended well-structured WF-net if and only if : (1) $P$ is a nonempty set of places, $T_{t}$ is a nonempty set of task transitions, $T_{b}$ is a set of block transitions and $T_{d}$ is a set of dummy task transitions with $P \cap\left(T_{t} \cup T_{b} \cup T_{d}\right)=$ $\varnothing, T_{t} \cap T_{b}=\varnothing T_{t} \cap T_{d}=\varnothing$ and $T_{b} \cap T_{d}=\varnothing$, and $F$ is called the set of flow relations with $F \subseteq\left\{P \times\left(T_{n} \cup T_{b} \cup T_{d}\right)\right\} \cup\left\{\left(T_{t} \cup T_{b} \cup T_{d}\right) \mathrm{x} P\right\}$; (2) $P N$ has two special places: $i$ and $o$. Place $i$ denotes the start of processes: $i=\varnothing$. Place $o$ means the end of processes: $o=\varnothing$; (3) the ret ynthesized by adding a transition $t^{*}$ to link $i$ with $o$ (i.e., $t^{*}=o$ and $t^{*}=i$ ) is strongly connected; (4) for any pair of nodes $x$ and $y$ of which one is a place and the other is a transition and for any pair of elementary paths $C_{1}$ and $C_{2}$ from $x$ to $y, C_{1} \cap C_{2}=(x, y)$ holas.

Business processes are driven by cases which in the paper are orders, and are accomplished by executing several tasks which are modeled as task transitions in $P N$. Building blocks AND-split (fork) and AND-join (join) are denoted as block transitions, while building blocks XOR-split (branch) and XOR-join (merge) are represented by places in $P N$. Each transition should start and end with a place. Only above mentioned building blocks are considered herein for other advanced blocks such as OR-split can be converted into the combinations of these four [24]. If two branches or merges are directly connected (e.g., branches 1 and 2 or merges 1 and 2 shown in Figure 2), a dummy task transition is inserted between them (e.g., dummy task transition 1 between branches 1 and 
2 or dummy task transition 2 between merges 1 and 2). Tokens in the places represent objects which in the paper are data units. The data flow passing through tasks in the business processes is represented by $\operatorname{arcs} F$ with arrows from places to transitions or from transitions to places. Flow patterns between tasks may be sequence, parallel split, synchronization, exclusive choice or simple merge, of which the latter four are respectively realized by fork, join, branch and merge. Tokens flow along the paths leading from $i$ to $o$, which denotes the completion of orders. In $P N$, the four building blocks are well balanced. That is, parallel flows initiated by the same fork (branch) should be joined by the same join (merge). Definition 1 makes business process modeling more formal, and business processes studied herein are supposed to be well-structured.

\subsection{Error Propagation}

Inadequate or failed execution of tasks in business processes will introduce different error types, and errors introduced at a task will propagate to its downstream tasks along data flow. Given that different flow patterns exist between tasks, error propagation between tasks can be realized in different paths with corresponding possibilities. An elementary path from $n_{1}$ to $n_{k}$ in $P N$ is a sequence $C=\left(n_{1}, n_{2}, \cdots, n_{k-1}, n_{k}\right)$ where $n_{i} \in P \cup$ $\left(T_{t} \cup T_{b} \cup T_{d}\right)$, arc $\left(n_{i}, n_{i+1}\right) \in F$ for $1 \leq i \leq k-1$ [11]. The following mentioned paths all refer to elementary paths. Tasks are disconnected if no path exist between them, otherwise they are connected. For connected tasks, two tasks are directly connected if no tasks lie on the paths between them, otherwise they are indirectly connected. Thus, error transition probability between any two task is either direc transition probability or indirect transition probability, and the transition probability between two disconnected tasks can be either direct or indirect.

Let $V=\left[v_{i j}\right]$ be the direct transition probability matrix where $v_{i j}$ is the probability that errors directly flow from tasks $i$ to , which is determined by the building block between them [4]. As shown in Figure 1, in the sequence pattern, error transition probability between tasks 1 and 2 is 1 (i.e., $v_{12}=1$ ) for only one path exists between them. In the exclusive choice pattern, ether task 2. or task 3 takes place after executing task 1. Data units output by task 1 flow either from task 1 to task 2 or to task 3 with probabilities $p$ and $1-p$. Thus, error transition probabilities from task 1 to tasks 2 and 3 are $p$ and $1-p$ (i.e., $v_{12}$ $=p, v_{13}=1-p$ ) For simple merge pattern, data go from task 1 or task 2 to task 3 , the transition probability between tasks 1 and 3 equals the probability between tasks 2 and 3 , with the value of $1\left(i, v_{3}=v_{23}=1\right)$. In the parallel split pattern, tasks 2 and 3 synchronize after finishing task 1 and data with errors output by task 1 flow to tasks 2 and 3 simultaneously. Therefore, error transition probabilities from task 1 to tasks 2 and 3 equal 1 (i.e., $v_{1}=v_{13}=1$ ). In synchronization pattern, errors flow from tasks 1 and 2 to task 3 with probabilities 1 (i.e., $v_{13}=v_{23}=1$ ). The reason is that task 3 starts after completing asks 1 and 2, and errors output by tasks 1 and 2 all flow to task 3 .

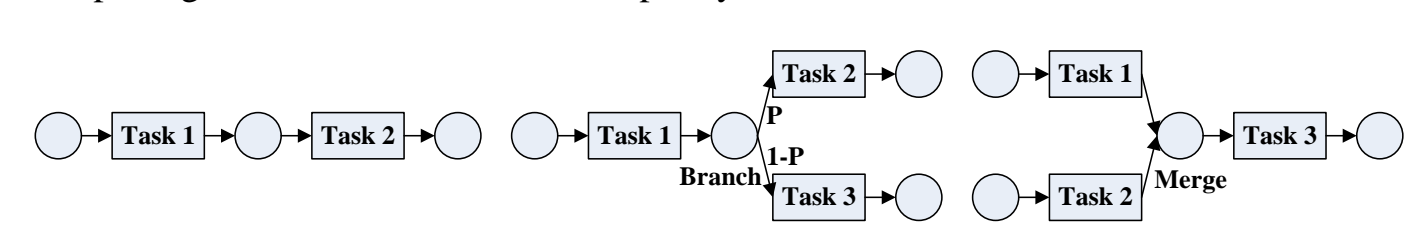

(a) Sequence

(b) Exclusive choice

(c) Simple merge

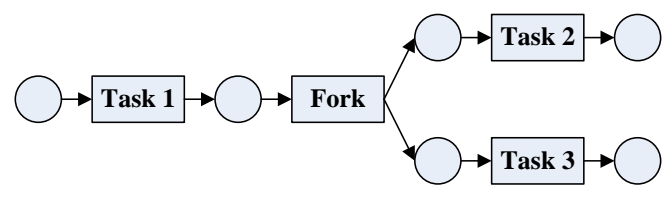

(d) Parallel split

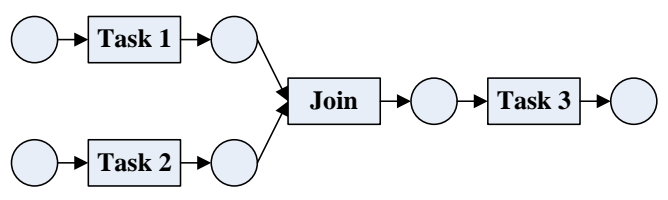

(e) Synchronization

\section{Figure 1. Five Basic Flow Patterns [24]}


Direct transition probability matrix $V$ is also called one-step transition probability matrix $V_{1}$. Transition probability between tasks $i$ and $j$ is called two-step transition probability if only task $k$ lies on the path between them, which equals the product of $v_{i k}$ and $v_{k j}$. If multiple such paths exist, transition probability between them is the sum of the probability of each path. Let $V_{2}$ denote the two-step transition probability matrix. By analogy, $V_{3}, V_{4}$, etc. can be obtained. Particularly, if there exist no paths between two tasks, the transition probability between them is 0 . Let $V_{k}(k \leq M-1)$ be the matrix with maximum step of business processes containing $M$ tasks. Error propagation matrix $E=\left[\gamma_{i j}\right]$ is defined as the sum of all transition probability matrices. The sum of the $i$ th column of $E$ (i.e., $\gamma_{+i}=\sum_{j=1}^{M} \gamma_{j i}$ ) represents the in-degree propagation potential of task $i$, which means that $\gamma_{+i}$ copies of errors will reach task $i$ if each of its upstream tasks introduces one copy of errors; the sum of $i$ th row (i.e., $\gamma_{i+}=\sum_{j=1}^{M} \gamma_{i j}$ ) denotes the out-degree propagation potential of task $i$, which signifies that $\gamma_{i+}$ copies of errors will reach its downstream tasks if task $i$ introduces one copy of errors [5].

\section{Robustness Optimization of Information Systems}

This section presents quantitative formulations of control effectiveness robustness on performance, robustness on cost and robustness on time based above formal model, and then establishes and analyzes the robustness optimization models.

\subsection{Control Effectiveness}

Resource such as manpower and equipment is jnput to handle errors after errors occurred, which is called a control. Effectiveness of control is the percentage of an error that the control can eliminate, and Bai et al. [5] define control effectiveness at task $i$ for error type $n$ as

$$
\bar{\alpha}_{i n}\left(x_{i n}\right)=k_{i n} \gamma_{+i} x_{i n}^{a_{i n}}
$$

The parameter $x_{i n}$ derotes the input level of resource at task $i$ for error type $n$ and equals the ratio of the actual number of resource input to all available resource. The exponent $a_{i n}$ is the marginal effectiveness of per unit input of resource. The parameter $\gamma_{+i}$ gatches the structural role of task $i$ on control effectiveness. The product $k_{i n}+i$ quantifies the maximal effectiveness that a control can achieve at task $i$ for error type $n$. The standardized factor $k_{i n}$ rescales the maximal effectiveness to the interval of $[0,1]$

In reality, the funetional role of task $i$ also affects control effectiveness. Ceteris paribus, the control applied at task $i$ with greater influence on system function is more effective than that at task $j$ with lower influence on system function, because resource such as human handles errors more careful spontaneously at task $i$ for its powerful influence on system function. Thus, control effectiveness at task $i$ for error type $n$ is rewritten as

$$
\alpha_{i n}\left(x_{i n}\right)=k_{i n} \gamma_{+i} \delta_{i} x_{i n}^{a_{i n}}
$$

The parameter $\delta_{i}$ represents the relative weight of task $i$ on system function which can be quantified as the residual closeness centrality [25].

\subsection{Robustness Measures}

Robustness in the paper is measured from dimensions of robustness on performance, robustness on cost and robustness on time.

\subsubsection{Robustness on Performance}

Under the perturbation of data errors, robust information systems still yield satisficing robustness on performance which is usually expressed as expected loss 
caused by errors. The smaller the expected loss is, the more robust the information systems are. Let $e_{i n}\left(x_{i n}\right)$ denote the loss per data unit caused by error type $n$ at task $i$ after control. The mathematical formulation of $e_{i n}\left(x_{i n}\right)$ is

$$
e_{\text {in }}\left(x_{\text {in }}\right)=l_{\text {in }} \gamma_{i+}\left(1-\alpha_{\text {in }}\right)
$$

The parameter $l_{\text {in }}$ represents the average loss per data unit of error type $n$ introduced by task $i$ that reaches its downstream tasks. Given that error type $n$ occurs at task $i$ with probability $p_{i n}$, the total expected loss $(i . e ., e)$ is given by

$$
e=\sum_{i, n} p_{i n} l_{i n} \gamma_{i+}\left(1-\alpha_{i n}\right)
$$

The smaller the value of $e$ is, the stronger the robustness on performance (i.e., $R_{p}$ ) is.

\subsubsection{Robustness on Cost}

It is intuitive that robust information systems can handle errors at a low cost. The cost is mainly determined by the wage or compensation for staff members and expenditure on technology and equipment. The lower the cost is, the more robust the information systems are. Let $\pi_{i n}\left(x_{i n}\right)$ denote the control cost per data anit of error type $n$ at task $i$. Bai et al. [5] define $\pi_{i n}\left(x_{i n}\right)$ as a Cobb-Douglas function

$$
\pi_{i n}\left(x_{i n}\right)=c_{i n} x_{i n}^{b_{i n}}
$$

The parameter $c_{i n}$ is the average control cost if all available resource is allocated at task $i$ for error type $n$. The exponent $b_{\text {in }}$ represents the marginal cost of per unit input of resource. Thus, the total control cost (i.e., $\pi$ ) is

$$
\pi=\sum_{i, n} \pi_{i n}\left(x_{i n}\right)
$$

The smaller the value of $\pi$ is, the stronge the robustness on cost $\left(i . e ., R_{c}\right)$ is.

\subsubsection{Robustness on Time}

Similarly, robust information systems can handle errors in a short time, and the time required equals the total time that all resource spends on disposing errors. The shorter the time is, the more robust the nformation systems are. Let $t_{i n}$ denote the average control time when all available resource is applied at task $i$ for error type $n$. Let control time be twice as large as $t_{i n}$ if no resource is input. Previous literature has illustrated that Cobb-Douglas functions are robust in various applications [26]. The control time per data unit at task $i$ for error type $n$ under the input level of resource $x_{i n}\left(i . e ., \omega_{i n}\left(x_{i n}\right)\right)$ is thus defined as

$$
\omega_{\text {in }}\left(x_{i n}\right)=2 t_{\text {in }}-x_{i n}
$$

The exponent $d$ neasures the time elasticity of resource input. Thus, the total control time (i.e., $\omega$ ) is expressed as

$$
\omega=\sum_{i, n} \omega_{i n}\left(x_{i n}\right)
$$

The smanler the value of $\omega$ is, the stronger the robustness on time (i.e., $\left.R_{t}\right)$ is.

\subsection{Robustness Optimization Models}

Robustness defined by Sawy et al. [21] reveals that it is worthwhile to pursue strong robustness on cost and robustness on time only when systems yield satisficing robustness on performance. However, formulas (6) and (8) disclose that systems cannot achieve strong robustness on cost and robustness on time simultaneously. This paper thus establishes two optimization models: the first is designed to achieve target robustness on performance with maximal robustness on cost, and the second is set to realize target robustness on performance with maximal robustness on time. 


\subsubsection{Maximize Robustness on Cost}

The robustness-on-cost-optimal model determines the optimal resource allocation policies by maximizing robustness on cost, and the model is specified as

$$
\begin{aligned}
& \mathrm{P}_{1}: \max R_{c} \\
& \text { s.t. } R_{p} \geq B ; \\
& 0 \leq x_{i n} \leq 1, \forall i, n .
\end{aligned}
$$

Lemma1. The optimal input level of resource at task $i$ for error type $n$ that maximizes robustness on cost and satisfies target robustness on performance is

$$
x_{i n / c}^{*}=\left(1 / \sum_{i, n} E_{i n}^{\frac{b_{i n}}{b_{i n}-a_{i n}}}\left(\frac{a_{i n}}{b_{i n} c_{i n}}\right)^{\frac{a_{i n}}{b_{i n}-a_{i n}}}\right)^{\frac{b_{i n}-a_{i n}}{a_{i n}}} \varphi_{i n}\left(\frac{1}{\sum_{i, n} p_{i n} l_{i n} \gamma_{i+}-B}\right)^{\varphi_{i n}}\left(\frac{a_{i n} k_{i n} p_{i n} l_{i n}}{b_{i n} c_{i n}}\right)^{\varphi_{i n}}\left(\gamma_{+i} \gamma_{i+} \delta_{i}\right)^{\varphi_{i n}}
$$

where $E_{\text {in }}=k_{i n} \gamma_{+i} \delta_{i} p_{i n} l_{i n} \gamma_{i+} /\left(\sum_{i, n} p_{i n} l_{\text {in }} \gamma_{i+}-B\right)$ and $\varphi_{\text {in }} \in\left\{0,1 /\left(b_{i n}-a_{i n}\right)\right\}$. Proof of lemma 1 is shown in appendix.

Suppose that parameters $k_{i n}, p_{i n}, l_{i n}, a_{i n}, b_{i n}$ and $c_{i n}$ are constant, then $x_{i n / c}^{*}$ is simplified as $x_{i n / c}^{*}=K_{1}\left(\gamma_{+i} \gamma_{i+} \delta_{i}\right)^{\varphi_{i n}}$ where $K_{1}$ represents the part containing these parameters, which reveals that more resource will be allocated to tasks with greater in-degree $\left(\gamma_{+i}\right)$ and out-degree $\left(\gamma_{i+}\right)$ propagation potentials and greater impact on system function $\left(\delta_{i}\right)$. Intuitively, a task with greater in-degree and out-degree propagation potentials receives and emits more errors errors occurred at a task with greater influence on system function impact system function to a larger extent. Such resource allocation policies detect and correct more errors, better prevent errors from propagation and better proteet systern tunction from errors by utilizing resources effectively, which is more likely to achieve target robustness on performance with maximal robustness on cost

\subsubsection{Maximize Robustness on Time}

Similarly, the robustness-on-time-optimization model finds the optimal resource allocation policies by maximizing yobustness on time, and the model is established as

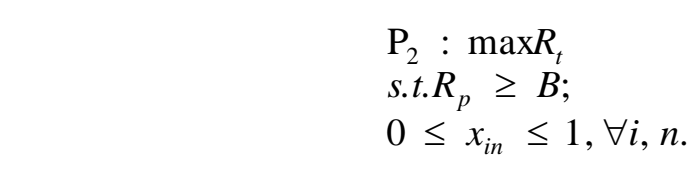

Lemma2. The optimal input level of resource at task $i$ for error type $n$ that maximizes robustness on time and satisfies target robustness on performance is $\left.x_{i n / t}^{*}=\sum_{i, n} E_{i n}^{\frac{d_{i n}}{d_{i n}-a_{i n}}}\left(\frac{a_{i n}}{d_{i n} t_{i n}}\right)^{\frac{a_{i n}}{d_{i n}-a_{i n}}}\right)^{\frac{d_{i n}-a_{i n}}{a_{i n}}}\left(\frac{1}{\sum_{i, n} p_{i n} l_{i n} \gamma_{i+}-B}\right)^{\phi_{i n}}\left(\frac{a_{i n} k_{i n} p_{i n} l_{i n}}{d_{i n} t_{i n}}\right)^{\phi_{i n}}\left(\gamma_{+i} \gamma_{i+} \delta_{i}\right)^{\phi_{i n}}$

where $E_{i n}=k_{i n} \gamma_{+i} \delta_{i} p_{i n} l_{i n} \gamma_{i+} /\left(\sum_{i, n} p_{i n} l_{i n} \gamma_{i+}-B\right)$ and $\phi_{i n} \in\left\{0,1 /\left(d_{i n}-a_{i n}\right)\right\}$. Proof of lemma 2 is also presented in appendix.

Analogously, parameters $k_{i n}, p_{i n}, l_{i n}, t_{i n}, a_{i n}$ and $d_{i n}$ are assumed to be constant, and $K_{2}$ represents the part containing these parameters. $x_{i n / t}^{*}$ is then altered into $x_{i n / t}^{*}=K_{2}\left(\gamma_{+i} \gamma_{i+} \delta_{i}\right)^{\phi_{i n}}$. The strategy to maximize robustness on time also configures more resource to tasks with bigger value of product $\gamma_{+i} \gamma_{i+} \delta_{i}$. Similarly, such resource 
allocation policies are most likely to achieve target robustness on performance with maximal robustness on time.

\section{Case Study}

This section validates the proposed methodology via a pharmaceutical enterprise in China. Figure 2 presents its order-fulfillment process using extended WF-net. The process includes 11 tasks, and the execution sequence is shown in Figure 2.

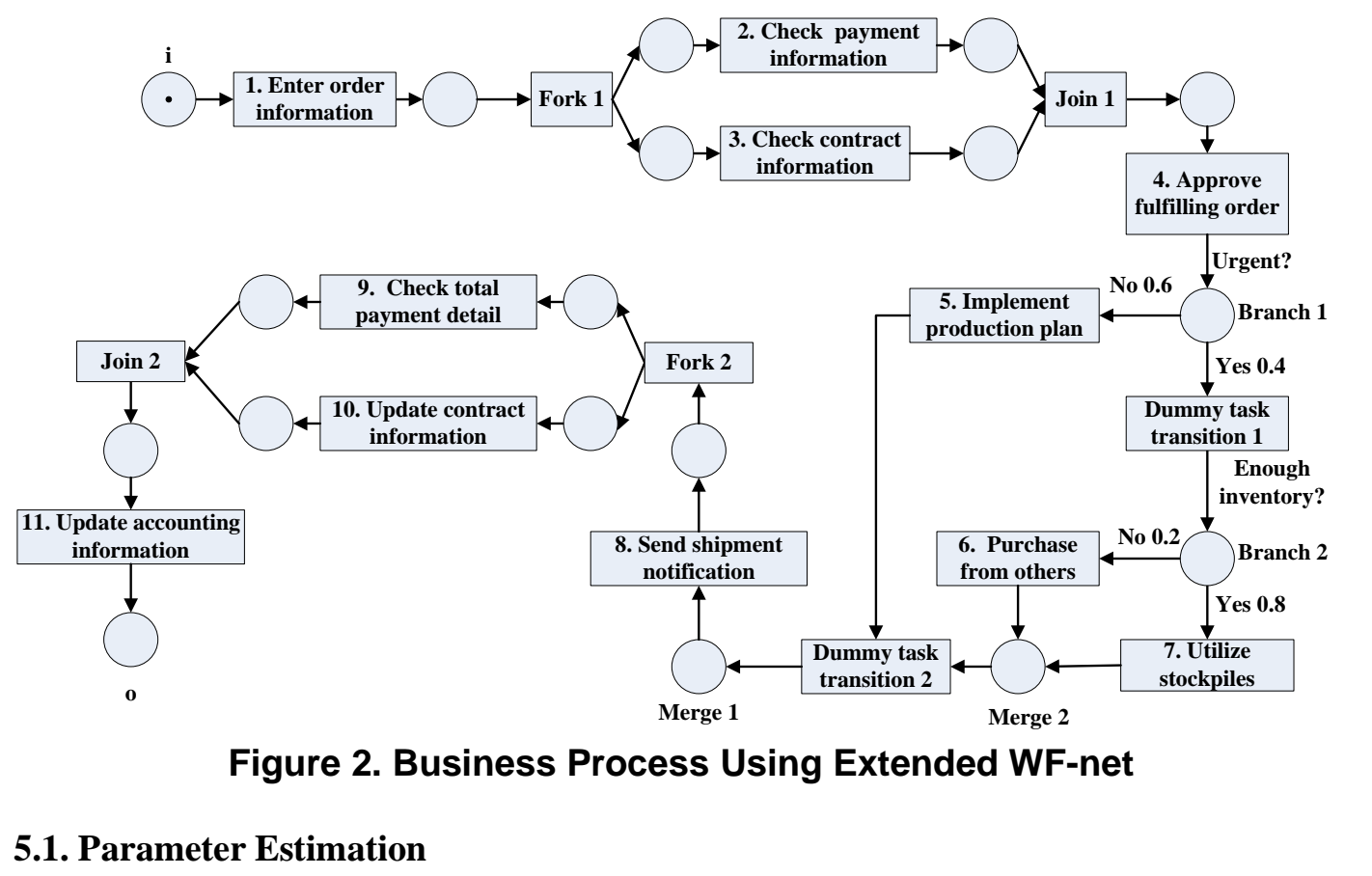

To illustrate the proposed methodology, the paper first provides parameter estimation. On average, the enterptise accomplishes 10000 orders yearly (i.e., 10000 data units), of which 4000 orders are urgent. Among the urgent orders, 3200 orders are completed by stockpiles and the remaining orders are finished via purchase. Thus, the ransition probabylities from task 4 to tasks 5,6 and 7 are $0.6,0.4 \times 0.2=$ 0.08 and $0.4 \times 0.8=0.32$ as shown in Figure 2. The rest transition probabilities between tasks omitted in Figure 2 all equal 1. Based on known transition probabilities, matrice $V$ and $E$ are worked out, as shown in Figure 3. The $i$ th row and column of both matrices represent task

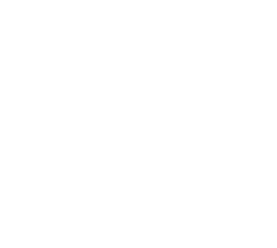

$$
V=\left[v_{i j}\right]=\left[\begin{array}{lllllllllll}
0 & 1 & 1 & 0 & 0 & 0 & 0 & 0 & 0 & 0 & 0 \\
0 & 0 & 0 & 1 & 0 & 0 & 0 & 0 & 0 & 0 & 0 \\
0 & 0 & 0 & 1 & 0 & 0 & 0 & 0 & 0 & 0 & 0 \\
0 & 0 & 0 & 0 & 0.6 & 0.08 & 0.32 & 0 & 0 & 0 & 0 \\
0 & 0 & 0 & 0 & 0 & 0 & 0 & 1 & 0 & 0 & 0 \\
0 & 0 & 0 & 0 & 0 & 0 & 0 & 1 & 0 & 0 & 0 \\
0 & 0 & 0 & 0 & 0 & 0 & 0 & 1 & 0 & 0 & 0 \\
0 & 0 & 0 & 0 & 0 & 0 & 0 & 0 & 1 & 1 & 0 \\
0 & 0 & 0 & 0 & 0 & 0 & 0 & 0 & 0 & 0 & 1 \\
0 & 0 & 0 & 0 & 0 & 0 & 0 & 0 & 0 & 0 & 1 \\
0 & 0 & 0 & 0 & 0 & 0 & 0 & 0 & 0 & 0 & 0
\end{array}\right]
$$




$$
E=\left[\gamma_{i j}\right]=\left[\begin{array}{llllllllllll}
0 & 1 & 1 & 2 & 1.2 & 0.16 & 0.64 & 2 & 2 & 2 & 4 \\
0 & 0 & 0 & 1 & 0.6 & 0.08 & 0.32 & 1 & 1 & 1 & 2 \\
0 & 0 & 0 & 1 & 0.6 & 0.08 & 0.32 & 1 & 1 & 1 & 2 \\
0 & 0 & 0 & 0 & 0.6 & 0.08 & 0.32 & 1 & 1 & 1 & 2 \\
0 & 0 & 0 & 0 & 0 & 0 & 0 & 1 & 1 & 1 & 2 \\
0 & 0 & 0 & 0 & 0 & 0 & 0 & 1 & 1 & 1 & 2 \\
0 & 0 & 0 & 0 & 0 & 0 & 0 & 1 & 1 & 1 & 2 \\
0 & 0 & 0 & 0 & 0 & 0 & 0 & 0 & 1 & 1 & 2 \\
0 & 0 & 0 & 0 & 0 & 0 & 0 & 0 & 0 & 0 & 1 \\
0 & 0 & 0 & 0 & 0 & 0 & 0 & 0 & 0 & 0 & 1 \\
0 & 0 & 0 & 0 & 0 & 0 & 0 & 0 & 0 & 0 & 0
\end{array}\right]
$$

Figure 3. Error Transition Probability Matrix and Error Propagation Matrix

The values for all $p_{\text {in }}$ are estimated by classifying 1336 error instances occurred in the 10000 orders across error types and tasks, and the values for all $l_{\text {in }}$ are estimated by the enterprise, as shown in Figure 4. For both matrices in Figure 4, the ithrow denotes task $i$, while the four columns represent accuracy errors, copipleteness errors, consistency errors and timeliness errors separately/For example, completeness errors occur at task 1 with probability $0.1 \%$, and sucherrors per order induce a loss of $100 \$$.

$$
P=\left[p_{i n}\right]=\left[\begin{array}{cccc}
2.5 \% & 0.1 \% & 0 & 0 \\
0.3 \% & 0 & 0 & 0 \\
0 & 0.1 \% & 0 & 0 \\
0 & 0 & 0.1 \% & 0 \\
0.03 \% & 0 & 0 & 0 \\
0.02 \% & 0 & 0 & 0 \\
0.3 \% & 0 & 0 \\
0 & 0 & 0.1 \% & 0 \\
9 \% & 0 & 0 & 0 \\
0 & 0.2 \% & 0 & 0 \\
0.1 \% & 0 & 0.5 \% & 0.01 \%
\end{array}\right]
$$

Figure 4. Error Probability Matrix and Loss Matrix

Numerical values of all $g_{i n}$ and $t_{i n}$ estimated by the enterprise are listed in Table 1. The values of all $\delta_{i}$ are computed by using software called CodeBlocks, and the result is also presented in Table 1 . The values of $a_{i n}, b_{i n}$ and $d_{i n}$ are estimated by taking the average across tasks and error types, and the values in the paper are $a_{i n}=$ $0.6(\forall i, n), b_{i n}=0.9(\forall i, n)$ and $d_{i n}=1(\forall i, n)$. The scale factor $k_{i n}(\forall i, n)$ is set as 0.35 to ensure that the product $k_{i n} \gamma_{+i} \delta_{i}$ is less than 1 for data errors cannot be eliminated. With the known parameters, the following section applies MATLAB to conduc a series of experiments.

\begin{tabular}{|c|c|c|c|c|c|c|c|c|c|c|c|}
\hline \multirow{2}{*}{ Parameter } & \multicolumn{11}{|c|}{ Task $i(\forall n)$} \\
\hline & 1 & 2 & 3 & 4 & 5 & 6 & 7 & 8 & 9 & 10 & 11 \\
\hline$c_{\text {in }}(\$)$ & 0.76 & 0.76 & 0.76 & 1.85 & 0.65 & 0.65 & 0.65 & 1.75 & 0.65 & 0.65 & 1.75 \\
\hline$t_{\text {in }}(\$)$ & 2.75 & 2 & 3.5 & 4 & 2 & 2 & 2 & 4 & 2 & 4 & 4.5 \\
\hline$\delta_{i}\left(10^{-2}\right)$ & 1.8 & 3.8 & 3.8 & 32.3 & 5.4 & 5.4 & 5.4 & 32.3 & 3.8 & 3.8 & 1.8 \\
\hline
\end{tabular}

Table 1. Estimation of $c_{i n}, t_{i n}$ and $\delta_{i}$

\subsection{Numerical Results and Analyses}

To analyze whether robustness on cost and robustness on time vary across control strategies and the values of target robustness on performance or not, section 5.2.1 
conducts three experiments under random control, robustness-on-cost-optimal control and robustness-on-time-optimal control respectively, and the values of target robustness on performance for the three experiments are achieving 1\%,50\% and $78 \%$ increment in the robustness on performance compared with the minimal robustness on performance. To analyze trends of robustness on cost and robustness on time as target robustness on performance increases, section 5.2.2 performs 242 experiments under the robustness-on-cost-optimal control and robustness-on-timeoptimal control separately, and the values of target robustness on performance for the 242 experiments are distributed uniformly in the interval of $[0,80 \%]$ where the element $x$ means $x$ percentage increment in the robustness on performance.

\subsubsection{Comparison of Robustness}

Table 2 proves that robustness on cost and robustness on time vary across control strategies and the values of target robustness on performance. More specifically, for $\mathrm{a}$ given target robustness on performance, the robustness on cost and robustness on time under the random control are worse than those under the other two controlstrategies; systems under the robustness-on-cost-optimal control posses $\$$ the strongest robustness on cost, but they are the most robust on time under the robustness-on-time-optimal control, which is intuitive. For a given control strategy, when arget robustness on performance increases, robustness on time increases but robustness on cost decreases. To explain above results, the input levels of resource across tasks and enror types under different control strategies are worked out, as shown in Figure 5.

Table 2. Robustness Comparison among Different Strategies

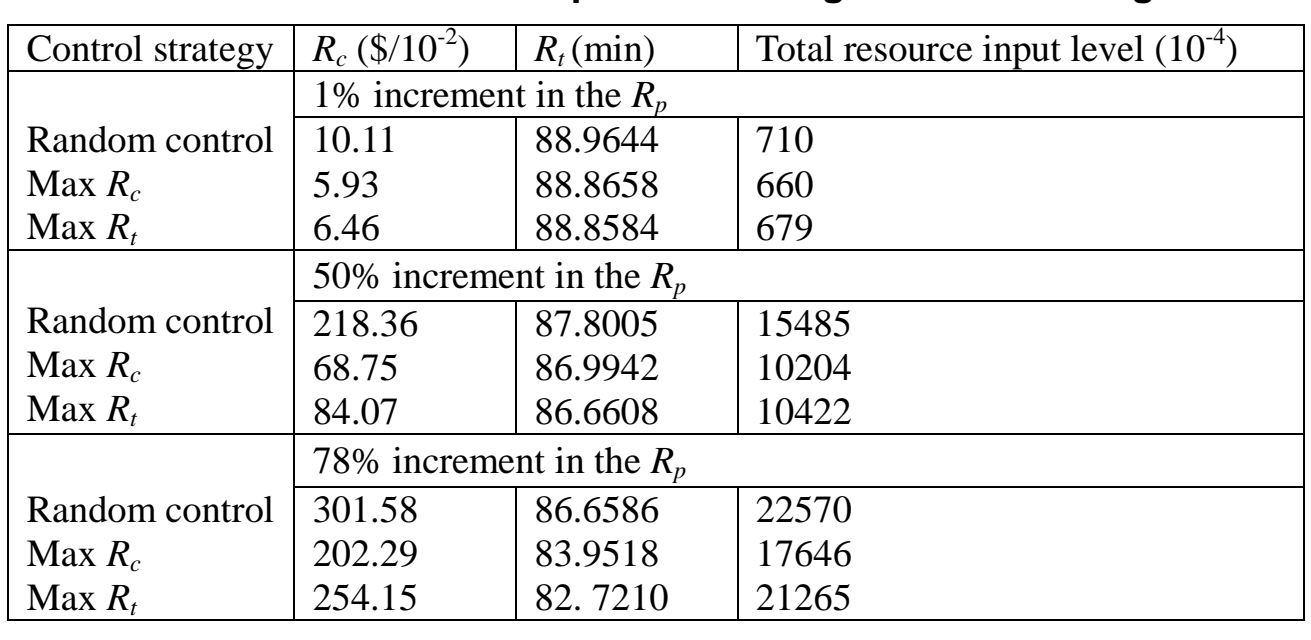

For a given target robustness on performance, Figure 5 shows that random control inputs resource randomly across tasks and error types, while robustness-on-time-optimal control inputs resource preferentially to the points characterized by high error propagation potentials $\left(\gamma_{+i} \gamma_{i+}\right)$, great influence on system function $\left(\delta_{i}\right)$, high error probability $\left(p_{i n}\right)$ or great loss $\left(l_{i n}\right)$. For example, the error probabilities of most points presented in Figure 4 (i.e., most $p_{i n}$ ) take the value of 0; random control still inputs resource at such points, whereas robustness-on-time-optimal control does not and inputs considerable resource at task 8 for consistency errors and task 9 for accuracy errors. The reason is that task 8 propagates the largest amount of errors (i.e., $\gamma_{+8} \gamma_{8+}=32$ is the biggest), consistency errors per order at task 8 impair system function to the largest extent and cause the hugest loss (i.e., the values of $\delta_{8}=0.323$ and $l_{83}=1000$ are bigger than those of other points), and task 9 introduces accuracy errors with the highest probability (i.e., $p_{91}=$ 9\% is the biggest).Thus, resource input at such tasks detects and corrects more errors, better prevents errors from propagation, or better protects system function from errors, 
which enables systems to resume normal operation in a shorter time and at a lower cost, that is, systems under the robustness-on-time-optimal control possess stronger robustness on time and robustness on cost than random control. The same pattern occurs when comparing random control with robustness-on-cost-optimal control. Moreover, Figure 5 shows that the input levels of resource across tasks and error types under the robustnesson-cost-optimal control are less than those under the robustness-on-time-optimal control, which accounts for the reality that the former control strategy yields better robustness on cost and worse robustness on time than the latter control strategy. For a given control strategy, Figure 5 indirectly illustrates that the input levels of resource across tasks increase when target robustness on performance increases, which explain the fact that increasing target robustness on performance cause decreasing robustness on cost and increasing robustness on time.

$\left[\begin{array}{cccc}34 & 3 & 21 & 67 \\ 25 & 24 & 28 & 21 \\ 53 & 22 & 43 & 17 \\ 5 & 1 & 3 & 4 \\ 22 & 10 & 26 & 5 \\ 11 & 6 & 13 & 6 \\ 39 & 7 & 38 & 26 \\ 8 & 7 & 2 & 4 \\ 33 & 17 & 2 & 34 \\ 3 & 4 & 3 & 8 \\ 1 & 2 & 1 & 1\end{array}\right]$

$\left(a_{1}\right)$ Random Control

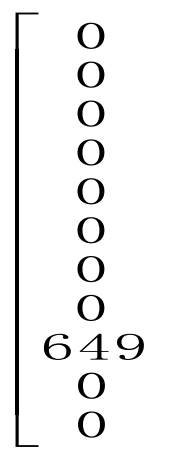

$\left(\mathrm{a}_{2}\right)$ Mâximize $R_{c}$

(a) $1 \%$ increment in the $B$

\section{$\left[\begin{array}{llll}515 & 48 & 318 & 1020\end{array}\right]$ $448 \quad 440508 \quad 386$ 1058440855341 $\begin{array}{llll}582 & 152 & 317 & 464\end{array}$ 385169452 \\ $\begin{array}{llll}170 & 91 & 195 & 89\end{array}$ 603114595400 $\begin{array}{lllll}755 & 668 & 18 & 407\end{array}$ $\begin{array}{lllll}396 & -199 & 27 & 407\end{array}$ $\begin{array}{ccccc}5 & 6 & 4 & 12 \\ 333 & 5 & 1 & 130 & 178\end{array}$}

$\left(b_{1}\right)$ Random Contro

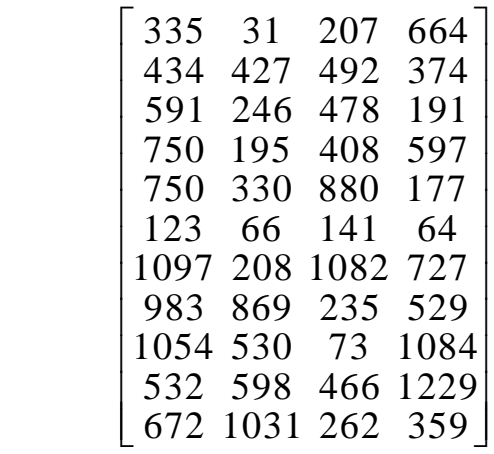

$\left(c_{1}\right)$ Random Control

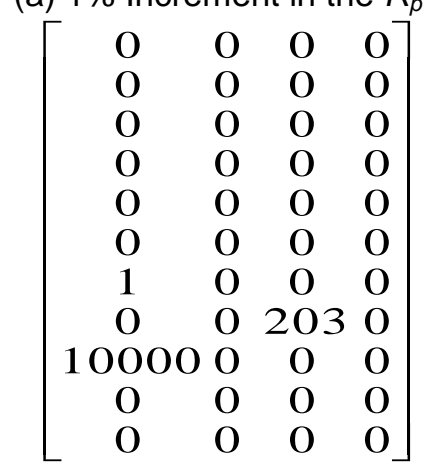

$\left(\mathrm{b}_{2}\right)$ Maximize $R_{c}$

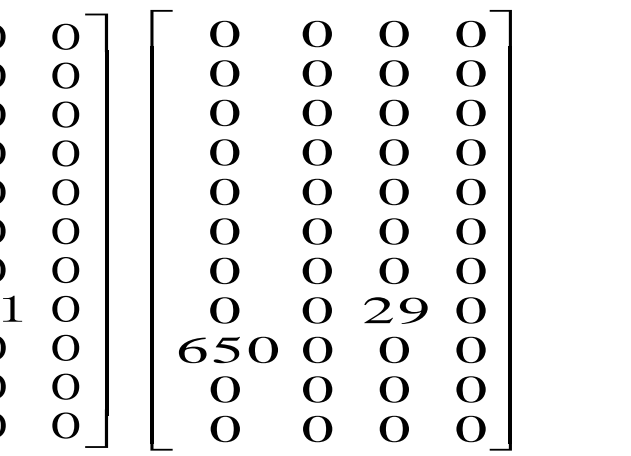

(a) Maximize $R_{t}$

(b) $50 \%$ Increment in the $R_{p}$

\begin{tabular}{|c|c|c|c|}
\hline $\mathrm{O}$ & $\mathrm{O}$ & O & 0 \\
\hline 3 & 0 & $\mathrm{O}$ & $\mathrm{O}$ \\
\hline $\mathrm{O}$ & 0 & $\mathrm{O}$ & U \\
\hline $\mathrm{O}$ & $\mathrm{O}$ & 1 & 0 \\
\hline O & O & O & $\mathrm{O}$ \\
\hline $\mathrm{O}$ & $\mathrm{O}$ & $\mathrm{O}$ & $\mathrm{O}$ \\
\hline 4 & $\mathrm{O}$ & $\mathrm{O}$ & 0 \\
\hline O & $\mathrm{O}$ & 414 & 0 \\
\hline 10000 & & O & 0 \\
\hline $\mathrm{O}$ & $\mathrm{O}$ & $\mathrm{O}$ & 0 \\
\hline $\mathrm{O}$ & 0 & 0 & 0 \\
\hline
\end{tabular}

$\left(\mathrm{b}_{3}\right)$ Maximize $R_{t}$

$\left[\begin{array}{cccc}0 & 0 & 0 & 0 \\ 14 & 0 & 0 & 0 \\ 0 & 0 & 0 & 0 \\ 0 & 0 & 1 & 0 \\ 0 & 0 & 0 & 0 \\ 0 & 0 & 0 & 0 \\ 37 & 0 & 0 & 0 \\ 0 & 0 & 7593 & 0 \\ 10000 & 0 & 0 & 0 \\ 0 & 1 & 0 & 0 \\ 0 & 0 & 0 & 0\end{array}\right]\left[\begin{array}{cccc}0 & 0 & 0 & 0 \\ 446 & 0 & 0 & 0 \\ 0 & 7 & 0 & 0 \\ 0 & 0 & 115 & 0 \\ 10 & 0 & 0 & 0 \\ 6 & 0 & 0 & 0 \\ 651 & 0 & 0 & 0 \\ 0 & 0 & 10000 & 0 \\ 10000 & 0 & 0 & 0 \\ 0 & 10 & 0 & 0 \\ 0 & 0 & 0 & 0\end{array}\right]$

$\left(\mathrm{c}_{2}\right)$ Maximize $R_{c}$

(c) $78 \%$ Increment in the $R_{p}$

Figure 5. Input Levels of Resource across Tasks and Error Types for a Given Target Robustness on Performance under Different Control Strategies $\left(10^{-4}\right)$ 


\subsubsection{Trend of Robustness}

As mentioned before, target robustness on performance is negatively correlated with robustness on cost and positively related with robustness on time. It is more interesting and important to find out the optimal target robustness on performance where robustness on cost and robustness on time do not change dramatically. To realize the target, 242 experiments are performed respectively under the robustness-on-cost-optimal control and robustness-on-time-optimal control. Figure 6 plots the change curves of robustness on cost and robustness on time as target robustness on performance increases. The optimal target robustness on performance under the robustness-on-cost-optimal control is achieving $76.7 \%$ percentage increment in robustness on performance, whereas under the robustness-on-time-optimal control is $75.6 \%$. Figure 6 shows that robustness on time is positively correlated with target robustness on performance - that is the good news, as the realization of stronger robustness on performance needs less time. Unfortunately, a substantially high cost is needed to satisfy the target robustness on performance exceeding the optimal value. For example, under both control strategies, if current target robustness on performance is optimal, then the cost is $2.4012 \$$ and $2.6000 \$$ under the tontrol strategies, and the time is $82.9986 \mathrm{mins}$ and $82.5901 \mathrm{mins}$. To achieve $80 \%$ increment in the robustness on performance, cost of $4.0223 \$$ and $6.3549 \$$ is required espectively, and the corresponding time needed is $78.6389 \mathrm{mins}$ and $70.8554 \mathrm{mms}$, which would decrease the robustness on cost by $67.5 \%$ and $144.4 \%$, yet increase the robustness on time only by $5.3 \%$ and $14.2 \%$. The conclusion from this observation is that it is unnecessary to input additional resource when target robustness on performance exceeds its optimal value. As to which control strategy to be adopted, it depends on the decision criteria of enterprises. For example, robustness-on-cost-optimal control is selected if enterprises purse strong robustness on cost and robustness on performance
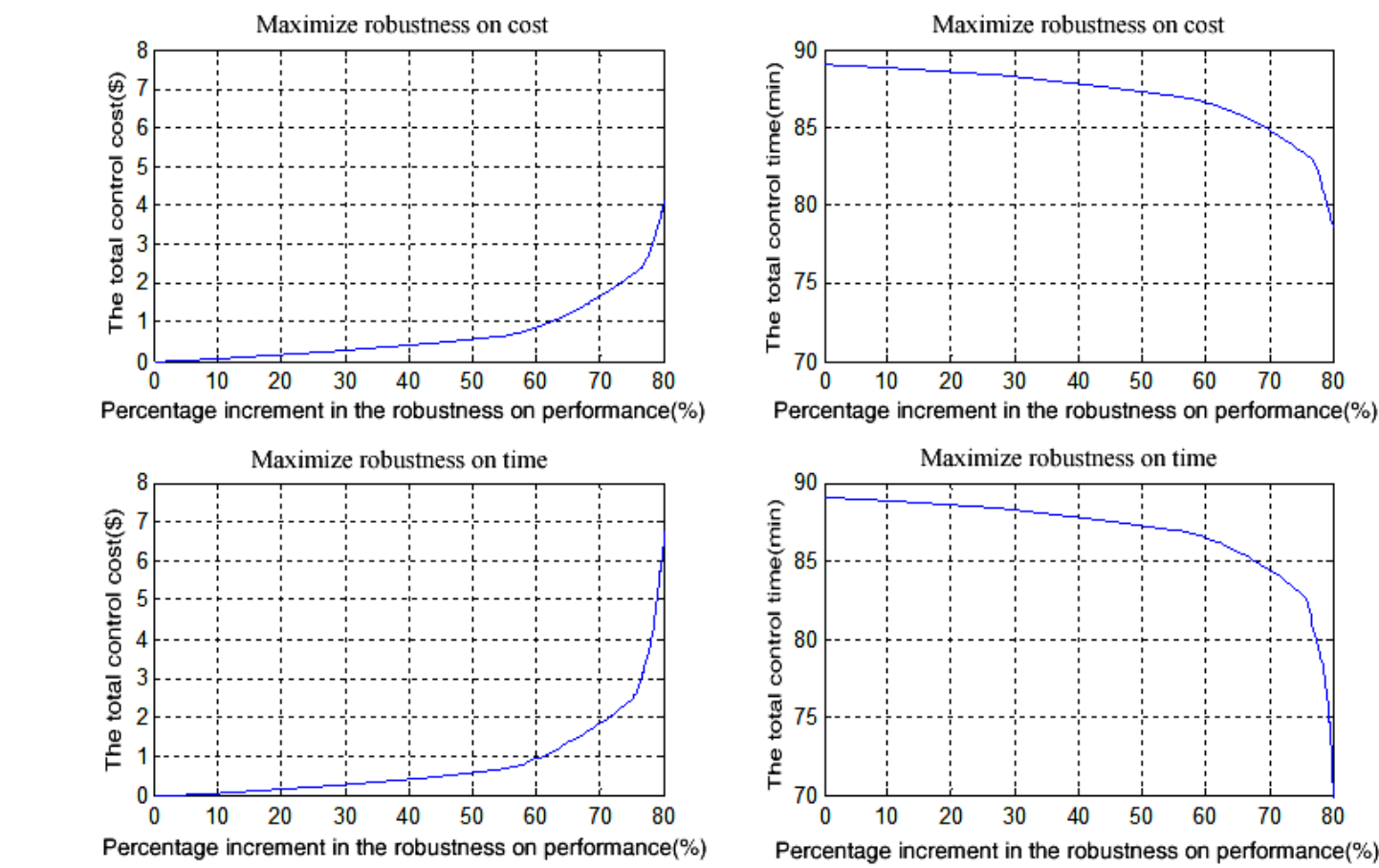

Figure 6. Trends of Robustness on Cost and Robustness on Time under the Two Control Strategies 


\section{Conclusions}

This paper has developed a process-oriented methodology for quantitative modeling of robustness management with data quality in information systems. This methodology extends well-structured WF-net to model information systems more formally, provides necessary elements to analyze robustness quantitatively, and establishes optimization models to find the optimal control policies that achieve target robustness on performance with maximal robustness on cost or robustness on time.

Results both from theoretical and numerical analysis yield several practical implications for robustness management with data quality of information systems. For system design, the research provides guide to design system that are robust to data errors through repeatedly adjusting the structural parameters $\gamma_{+i}$ and $\gamma_{i+}$ and the functional parameter $\delta_{i}$ in numerical simulation. For system operation, this study provides feasible resource allocation policies that achieve satisfying robustness after data errors occurred. As to which resource allocation policy to be adopted, it depends on the decision eriteria of enterprises (e.g., control cost, control time and performance). In addition, improving parameters $p_{i n}, l_{i n}, c_{i n}$, etc. before occurrence of errors is an effective feed-forward control strategy to increasing system robustness.

This study has two main limitations. First, the proposed optrmization models are static, while parameters such as $c_{i n}$ may change over time. In this situation, the availability of the proposed models will be greatly impaired. Second the disruptive factor considered herein is data quality. In fact, many other factors (e.g., unreliable resource) can influence system robustness. The robustness management under several factors is more complex and difficult, and the proposed methodology hus cannot be directly applied. Future work includes developing dynamic policies and establishing robustness-optimization models incorporating several factors for robustnes management of information systems.

\section{Appendix}

\section{Proof of Lemma1}

The objective to maximize robustness on cost equals the objective to minimize total control cost, and to achieve robustness on performance exceeding target $B$ equals to make expected loss-under the target $B$, that is,

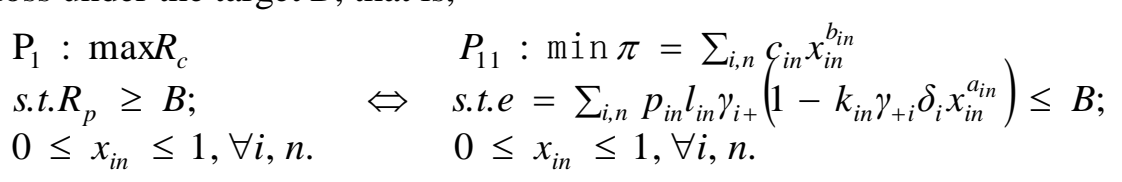

Let $E_{i n}=k_{i n} \gamma_{+i} \delta_{i} p_{i n} l_{i n} \gamma_{i+} /\left(\sum_{i, n} p_{i n} l_{i n} \gamma_{i+}-B\right) ; \rho_{i n}=x_{i n}^{a_{i n}} ; \theta=b_{i n} / a_{i n} . \mathrm{P}_{11}$ is transformed as,

$$
\begin{aligned}
& \mathrm{P}_{12}: \min R_{c}=\sum_{i, n} c_{i n} \rho_{i n}^{\theta_{i n}} \\
& \text { s.t. } \sum_{i, n} E_{i n} \rho_{i n} \geq 1 ; \\
& 0 \leq \rho_{\text {in }} \leq 1, \forall i, n .
\end{aligned}
$$

The Kuhn-Tucker conditions of $\mathrm{P}_{12}$ are

$$
\left\{\begin{array}{l}
\nabla \sum_{i, n} c_{i n} \rho_{i n}^{\theta_{i n}}-\lambda \nabla\left(E_{i n} \rho_{\text {in }}-1\right)-u \nabla \rho_{\text {in }}-\eta \nabla\left(1-\rho_{\text {in }}\right)=0 \\
\lambda\left(E_{\text {in }} \rho_{\text {in }}-1\right)=0 ; u \rho_{\text {in }}=0 ; \eta\left(1-\rho_{\text {in }}\right)=0 ; \lambda, u, \eta \geq 0 .
\end{array}\right.
$$

The Kuhn-Tucker point that satisfies above conditions is 


$$
\rho_{\text {in/c }}^{*}=\left\{\begin{array}{c}
\left(\frac{\lambda E_{\text {in }}}{\theta c_{\text {in }}}\right)^{\frac{1}{\theta-1}},(\mu=0 \wedge \eta=0) \wedge\left(\left(\frac{\lambda E_{\text {in }}}{\theta c_{\text {in }}}<1 \wedge \theta>1\right) \vee\left(\frac{\lambda E_{\text {in }}}{\theta c_{\text {in }}}>1 \wedge \theta<1\right)\right) \wedge \lambda=\left(1 / E_{\text {in }}^{\frac{\theta}{\theta-1}}\left(\frac{1}{\theta c_{\text {in }}}\right)^{\frac{1}{\theta-1}}\right)^{\theta-1} \\
1 \quad,\left(\theta c_{i n}-\lambda E_{\text {in }}+\eta=0 \wedge \mu=0 \wedge \sum_{i, n} E_{\text {in }}=1\right) \vee\left(\eta=\theta c_{\text {in }} \wedge \lambda=0 \wedge \mu=0\right)
\end{array}\right.
$$

The optimal solution of problem $\mathrm{P}_{1}$ is

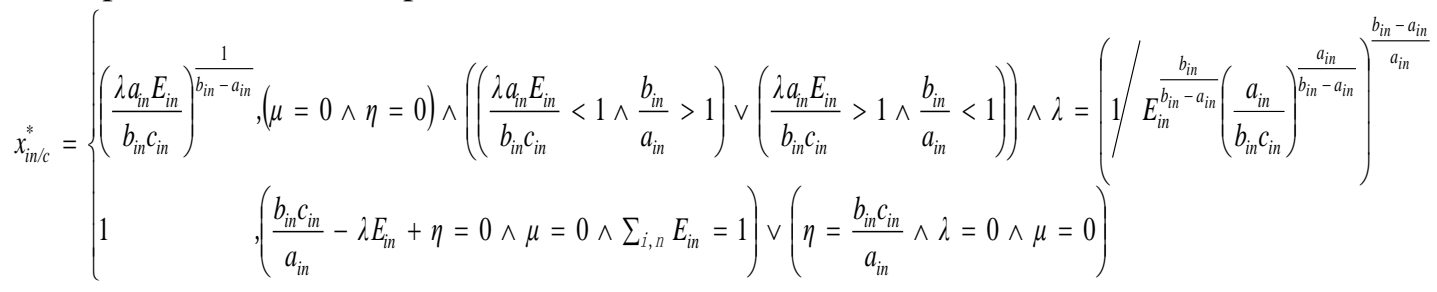

Given that $\left(\lambda a_{i n} E_{i n} / b_{i n} c_{i n}\right)^{0}=1, x_{i n / c}^{*}$ can be unified as $x_{i n / c}^{*}=\left(\lambda a_{i n} E_{i n} / b_{i n} c_{i n}\right)^{\varphi_{i n}}$ where

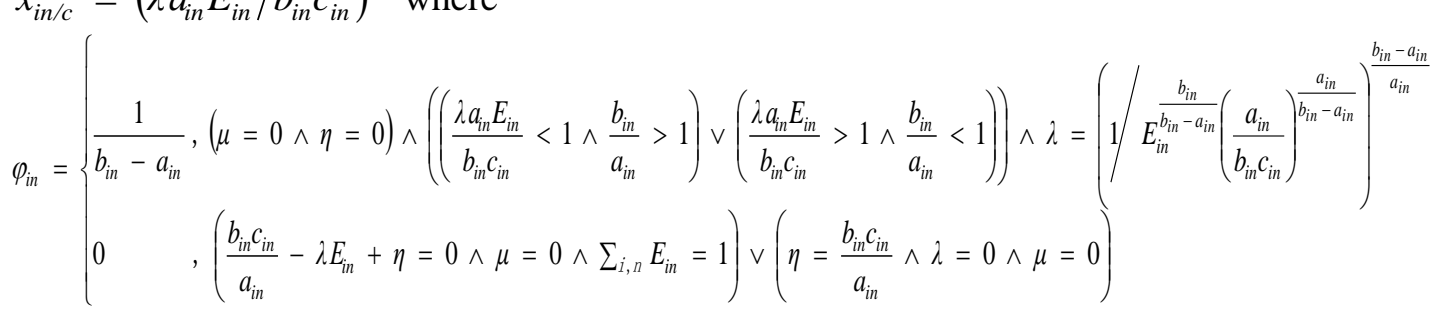

\section{Proof of Lemma2}

The objective to maximize fobusthess on time equals the objective to minimize total control time, and to achieve robustness on performance exceeding the $\operatorname{target} B$ equals to make expected loss under the target $B$, that is,

$$
\begin{aligned}
& \mathrm{P}_{2}: \max R_{t} \\
& \text { s.t. } R_{p} \geq B \\
& 0 \leq x_{i} \leq 1, \forall i, n .
\end{aligned} \Leftrightarrow \begin{aligned}
& P_{2} . \min \omega=\sum_{i, n} 2 t_{i n}-t_{i n} x_{i n} \\
& \text { s.t.e }=\sum_{i, n} p_{i n} l_{i n} \gamma_{i+} \\
& 0 \leq x_{i n} \leq 1, \forall i, n .
\end{aligned}
$$

Let $E_{\text {in }}=k_{i n} \gamma_{+i} \delta_{i} p_{i n} l_{\text {in }} P\left(\sum_{i, n} p_{i n} l_{i n} \gamma_{i+}-B\right) ; \rho_{i n}=x_{i n}^{a_{i n}} ; \sigma=d_{i n} / a_{i n} . \mathrm{P}_{21}$ is transformed as

The Kahn,Tucker conditions of $\mathrm{P}_{12}$ are

$$
\begin{aligned}
& \mathrm{P}_{22}: \min R_{t}=\sum_{i, n} 2 t_{i n}-t_{i n} \rho_{i n}^{\sigma} \\
& \text { s.t. } \sum_{i, n} E_{\text {in }} \rho_{\text {in }} \geq 1 ; \\
& 0 \leq \rho_{\text {in }} \leq 1, \forall i, n .
\end{aligned}
$$

$$
\left\{\begin{array}{l}
\nabla \sum_{i, n} 2 t_{\text {in }}-t_{\text {in }} \rho_{\text {in }}^{\theta_{\text {in }}}-\beta \nabla\left(E_{\text {in }} \rho_{\text {in }}-1\right)-\varepsilon \nabla \rho_{\text {in }}-\tau \nabla\left(1-\rho_{\text {in }}\right)=0 \\
\beta\left(E_{\text {in }} \rho_{\text {in }}-1\right)=0 ; \varepsilon \rho_{\text {in }}=0 ; \tau\left(1-\rho_{\text {in }}\right)=0 ; \beta, \varepsilon, \tau \geq 0 .
\end{array}\right.
$$

The Kuhn-Tucker point meeting above conditions is

$\rho_{i n / t}^{*}=1,\left(-\sigma_{i n}-\beta E_{i n}+\tau=0 \wedge \varepsilon=0 \wedge \sum_{i, n} E_{i n}=1\right) \vee\left(\tau=\sigma_{i n} \wedge \beta=0 \wedge \varepsilon=0\right)$

In the case that $\beta \neq 0$ and $\varepsilon=\tau=0$, Kuhn-Tucker formulation is ineffective to find out the optimal solution. The case of $\beta \neq 0$ and $\varepsilon=\tau=0$ indicates that $\sum_{i, n} E_{i n} \rho_{i n}$ strictly equals 1 and the value of $\rho_{i n}$ is within the interval of $(0,1) . \mathrm{P}_{22}$ is rewritten as 


$$
\begin{aligned}
& \mathrm{P}_{23}: \min R_{t}=\sum_{i, n} 2 t_{i n}-t_{i n} \rho_{i n}^{\sigma} \\
& \text { s.t. } \sum_{i, n} E_{\text {in }} \rho_{\text {in }}=1 ; \\
& 0<\rho_{\text {in }}<1, \forall i, n .
\end{aligned}
$$

The Lagrangian formulation of $\mathrm{P}_{23}$ is

$$
L\left(\rho_{i n}, \beta\right)=\sum_{i, n} 2 t_{i n}-t_{i n} \rho_{i n}^{\sigma}+\beta\left(\sum_{i, n} E_{i n} \rho_{i n}-1\right)
$$

The Lagrangian conditions of $\mathrm{P}_{23}$ are

$$
\left\{\begin{array}{l}
\frac{\partial L}{\partial \rho_{i n}}=-\sigma t_{i n} \rho_{i n}^{\sigma-1}+\beta E_{i n}=0 \\
\frac{\partial L}{\partial \beta}=\sum_{i, n} E_{i n} \rho_{i n}-1=0 .
\end{array}\right.
$$

The optimal solution of $\mathrm{P}_{23}$ is

$\rho_{\text {int }}^{*}=\left(\frac{\beta E_{i n}}{\sigma_{i n}}\right)^{\frac{1}{\sigma-1}},(\varepsilon=0 \wedge \tau=0) \wedge\left(\left(\frac{\beta E_{i n}}{\sigma_{i n}}<1 \wedge \sigma>1\right) \vee\left(\frac{\beta E_{i n}}{\sigma \sigma_{i n}}>1 \wedge \sigma<1\right)\right) \wedge \beta \Rightarrow\left(E_{i n}^{\sigma}\left(\frac{1}{\sigma_{i n}}\right)^{\frac{1}{\sigma-1}}\right)^{\sigma-1}$

Thus, the optimal solution of $\mathrm{P}_{21}$ is

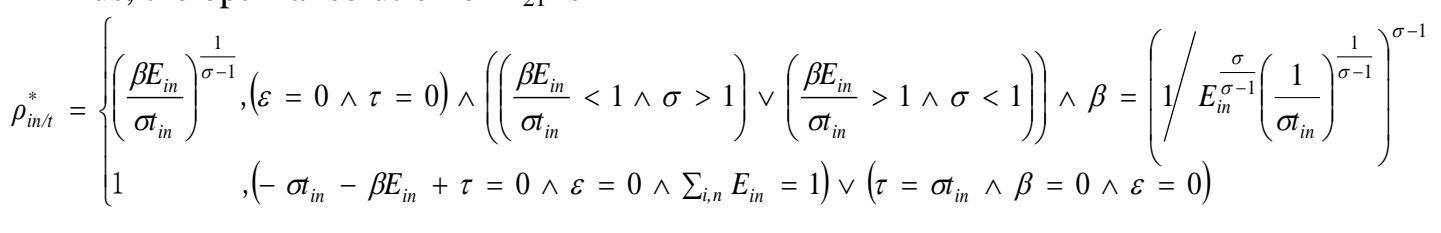

The optimal solution of $\mathrm{P}_{2}$ is

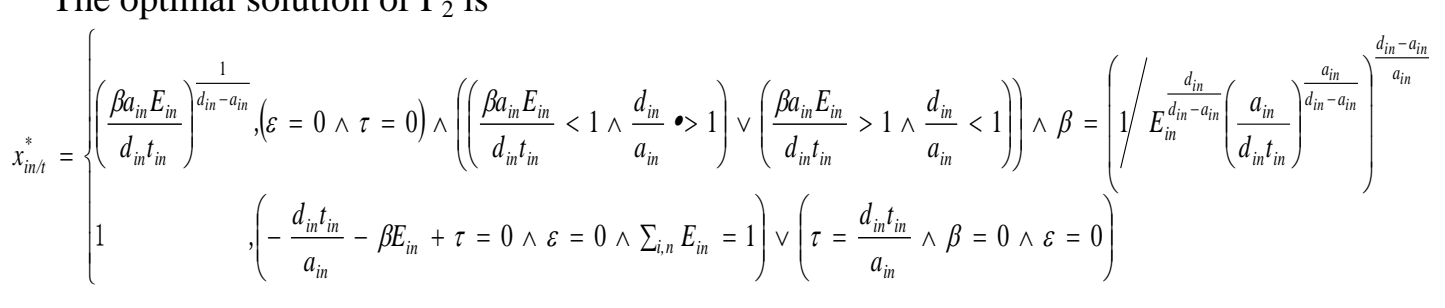

Given that $\left(\beta a_{i n} E_{\text {in }}\left(\mathcal{O}_{\text {in }} t_{\text {in }}\right)^{0}=1, x_{\text {in/t }}^{*}\right.$ can be unified as $x_{i n / t}^{*}=\left(\beta a_{i n} E_{\text {in }} / d_{\text {in }} t_{\text {in }}\right)^{\phi_{i n}}$ where

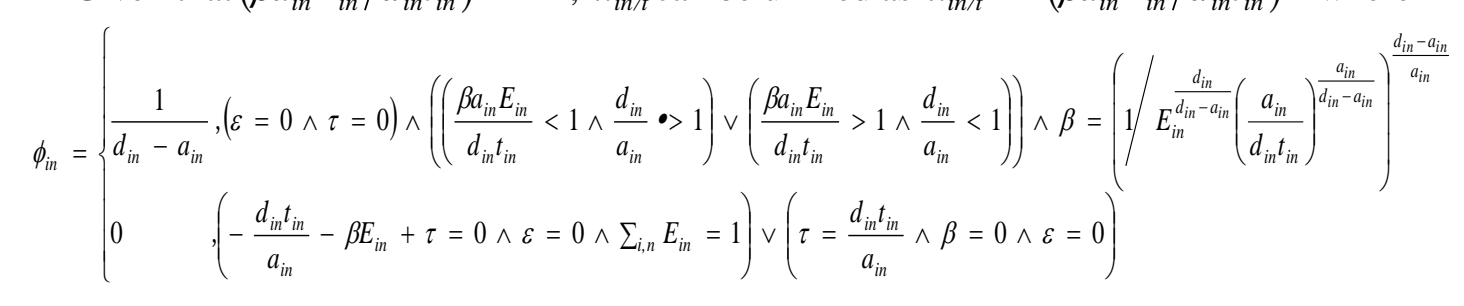

\section{Acknowledgements}

This study is supported by National Natural Science Foundation of China (No. 71571072, 50675069, 71071059). 


\section{References}

[1] M. L. Brodie, "Data Quality in Information Systems”, Information \& Management, vol. 3, no. 6, (1980), pp. 245-258.

[2] R. Krishnan, J. Peters, R. Padman and D. Kaplan, "On Data Reliability Assessment in Accounting Information Systems", Information Systems Research, vol. 16, no. 3, (2005), pp. 307-326.

[3] B. Heinrich and M. Klier, "Metric-Based Data Quality Assessment-Developing and Evaluating a Probability-Based Currency Metric”, Decision Support Systems, vol. 72, (2015), pp. 82-96.

[4] X. Bai, M. Nunez and J. R. Kalagnanam, "Managing Data Quality Risk in Accounting Information Systems", Information Systems Research, vol. 23, no. 2, (2012), pp. 453-473.

[5] X. Bai, R. Krishnan, R. Padman and H. J. Wang, "On Risk Management with Information Flows in Business Processes”, Information Systems Research, vol. 24, no. 3, (2013), pp. 731-749.

[6] Y. Wand and R. Y. Wang, "Anchoring Data Quality Dimensions in Ontological Foundations", Communications of the ACM, vol. 39, no. 11, (1996), pp. 86-95.

[7] R. Y. Wang, V. C. Storey and C. P. Firth, “A Framework for Analysis of Data Quality Research”, IEEE Transaction on Knowledge \& Data Engineering, vol. 7, no. 4, (1995), pp. 623-640.

[8] R. Y. Wang and D. M. Strong, "Beyond Accuracy: What Data Quality Means to Data Consumers", Journal of Management Information Systems, vol. 12, no. 4, (1996), pp. 5-33.

[9] D.M. Strong, Y. W. Lee and R. Y. Wang, "Data Quality in Context", Communications of the ACM, vol. 40, no. 5, (1997), pp. 103-110.

[10] R. B. Lea, S. J. Adams, R. F. Boykin and W. Smieliauskas, "Modeling of the Audit Risk Assessment Process at the Assertion Level within an Account Balance", Auditing, vol. 11(Supplement), (1992), pp. $152-179$

[11] W. M. P. Van der Aalst, "The Application of Petri Nets to Wôkflow Management" Journal of Circuits, Systems \& Computers, vol. 8, no. 1, (1998), pp. 21-66.

[12] R.S. Aguilar-Savén, "Business Process Modelling: Review and Framework", International Journal of Production Economics, vol. 90, no. 2, (2004), pp. $122-149$.

[13] W. Sadiq and M. E. Orlowska, "Analyzing Prôcess Models Using Graph Reduction Techniques", Information Systems, vol. 25, no. 2, (2000), pp (117-134.

[14] A. W. Scheer, "ARIS-Business Process Modeling", Springer Berlin, (2000).

[15] D. Liu, J. Wang, S. C. F. Chan, J. Sun and I.Zhang, "Modeling Workflow Processes with Colored Petri Nets", Computers in Industry, vol. 49, no 3, (2002), pp 267-281.

[16] K. Salimifard and M. Wright, "Petr Net-Based Modelling of Workflow Systems: An Overview", European Journal of Operational Research, vol 134 no. 3, (2001), pp. 664-676.

[17] M. Tavana, D. A. Trevisani and L. Dussault, "The Stability Model: An Interactive Framework for Measuring Robustness and Resiliency in Military Command and Control Systems", International Journal of Information Te linollogy Project Management, vol. 4, no. 2, (2013), pp. 18-34.

[18] S. D. Gribble, "Robustness in Complex Systems", Proceedings of the Eighth Workshop on Hot Topics in Operating Systems, Elmau, Germany, (2001) May 20-22.

[19] R. Malak, B. Baxter and C. Hsia, A Decision-Based Perspective on Assessing System Robustness", Procedia Computer Science, vol. 44, (2015), pp. 619-629.

[20] M. Shah, "Performance a P Measure of Robustness", Structures Congress 2009: Don't Mess with Structura Engineers: Expanding Our Role, Austin, Texas, (2009), April 30-May 2.

[21] O. A. E. Sawy and B. Nanus, "Toward the Design of Robust Information Systems", Journal of Management Information Systems, vol. 5, no. 4, (1989), pp. 33-54.

[22] N. Stricker and G. Lanza, "The Concept of Robustness in Production Systems and Its Correlation to Disturbances Procedia CIRP, vol. 19, (2014), pp. 87-92.

[23] M. Tavana, D. A. Trevisani and J. L. Dussault, "The Fuzzy Stability Model: An Interactive Framework for Measuring Robustness and Resiliency under Uncertainty", International Journal of Data Analysis Techniques \& Strategies, vol. 6, no. 2, (2014), pp. 137-161.

[24] W. M. P. Van der Aalst, A. H. M. Ter Hofstede, B. Kiepuszewski and A. P. Barros, "Workflow Patterns", Distributed \& Parallel Databases, vol. 14, no. 1, (2003), pp. 5-51.

[25] C. Dangalchev, "Residual Closeness in Networks", Physica A: Statistical Mechanics \& Its Applications, vol. 365 , no. 2 , (2006), pp. 556-564

[26] C.W. Cobb and P. H. Douglas, "A Theory of Production", The American Economic Review, vol. 18, no. 1, (1928), pp. 139-165. 


\section{Authors}

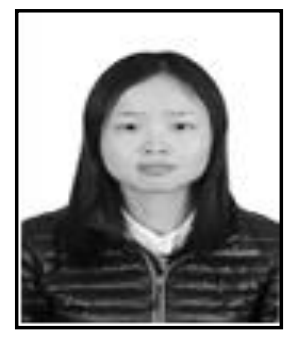

Zhiting Song, She is currently a doctoral candidate in the school of business administration of South China University of Technology. Her areas of interest are information systems, service-oriented manufacturing, and complex systems.

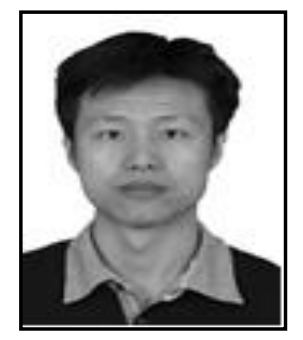

Yanming Sun, He is currently a professor and doctoral supervisor in the school of business administration of South China University of Technology. His areas of interest are manufacturing systems, information systems, and system simulation.

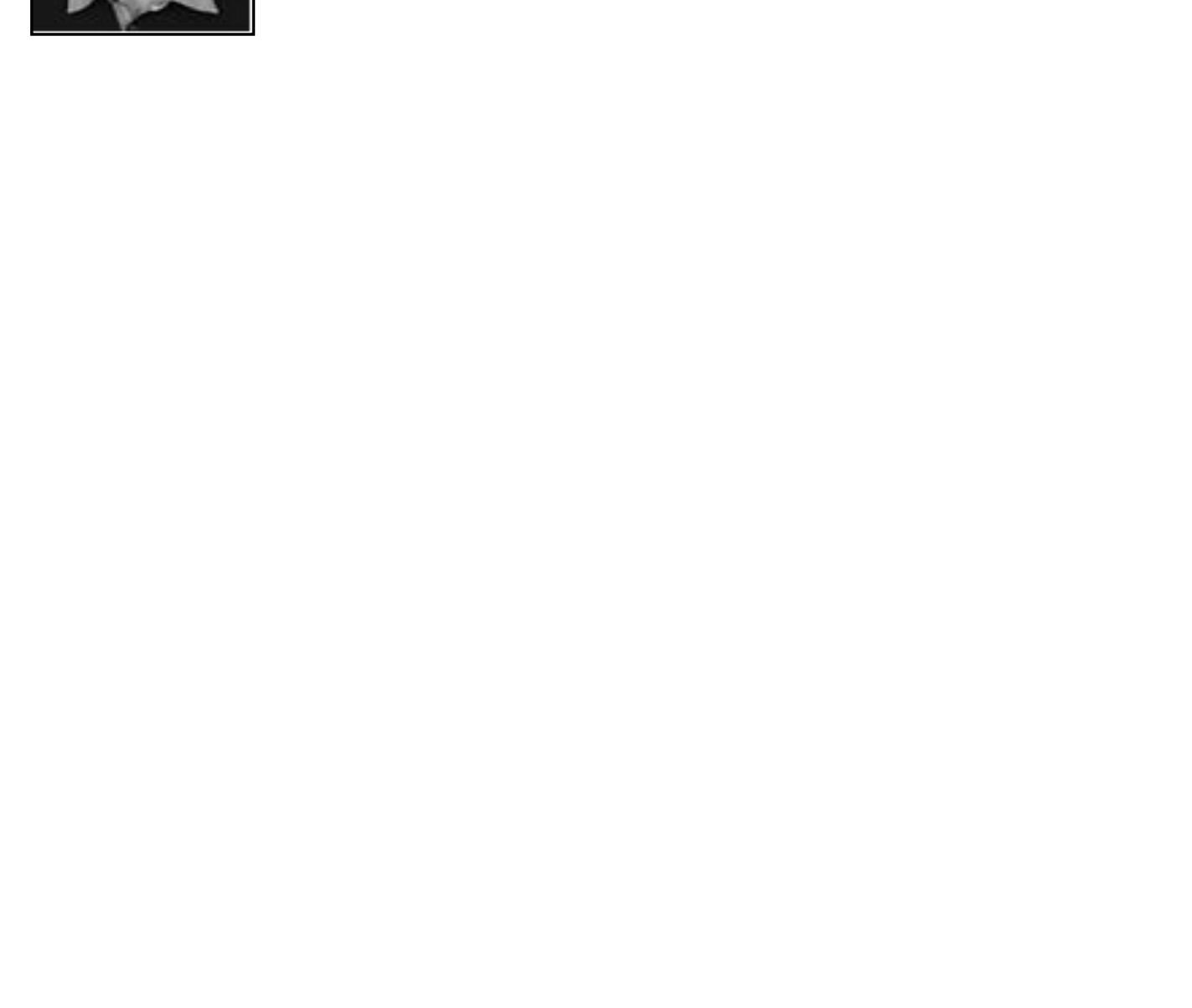


International Journal of Hybrid Information Technology

Vol. 9, No.9 (2016)

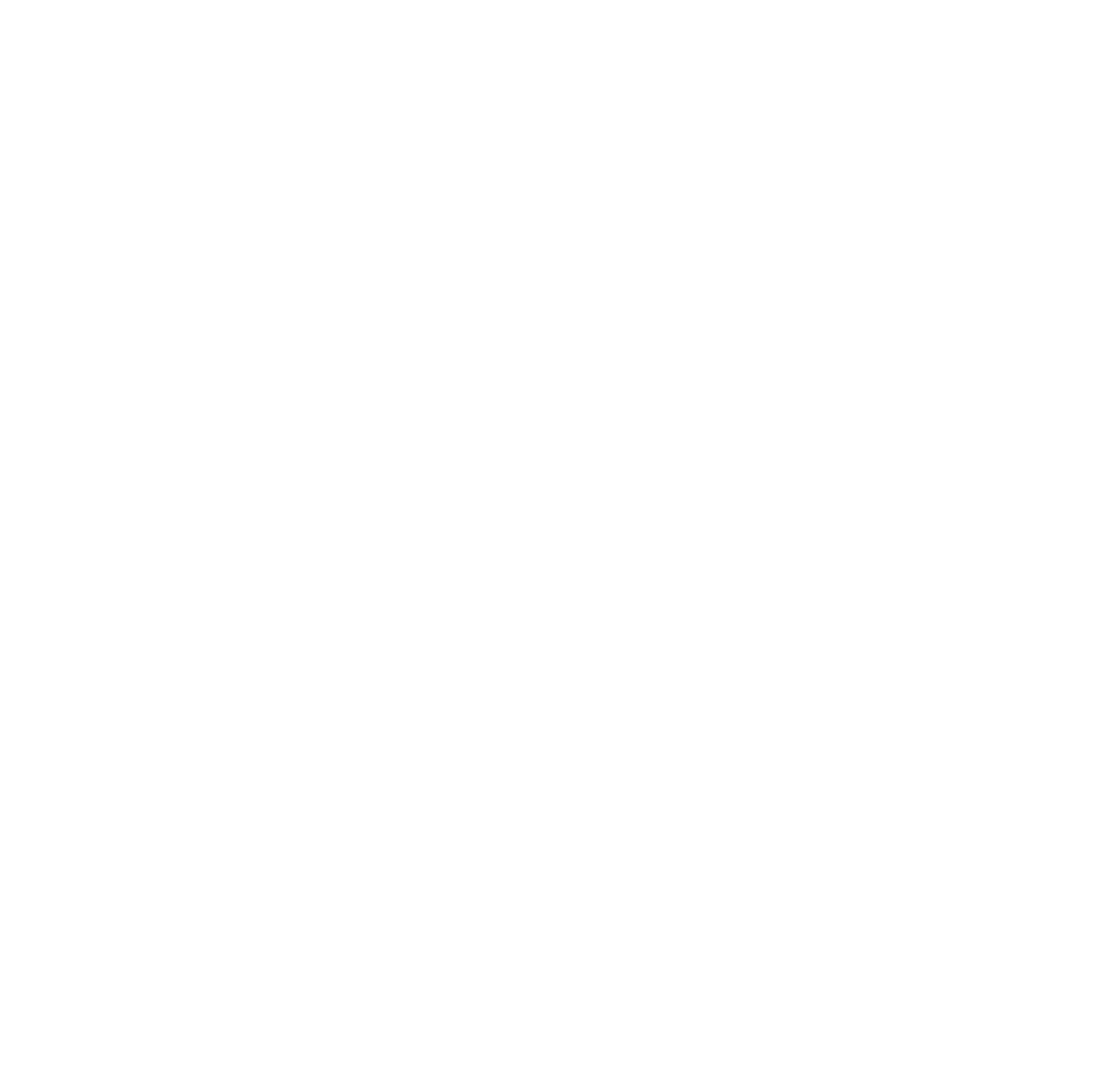

